\title{
La lógica de las grandes acciones inmobiliarias en la ciudad de Querétaro*
}

\author{
Beatriz García Peralta**
}

Este artículo analiza aspectos relevantes de la actividad inmobiliaria realizada en una ciudad media mexicana (Querétaro) durante los años 1960-1982. En dicho periodo se dio un importante crecimiento urbano en la localidad, relacionado con su desarrollo industrial.

En el trabajo se analizan tres operaciones inmobiliarias de la localidad que, por su extensión y ubicación, se convirtieron en las más significativas. Entre otros aspectos, los resultados de esta investigación muestran que los desarrollos inmobiliarios fueron llevados a cabo por grupos que concentraron y efectuaron la totalidad de las actividades que implica el proceso de urbanización y circulación de la tierra, así como de la producción de vivienda. También resalta la facilidad de acceso, por parte de estos grupos, a grandes extensiones de tierra privada agrícola para destinarla a usos urbanos. Aunque estas características de la actividad inmobiliaria también se dan en otras localidades del país, adquieren una dimensión particular en el caso de Querétaro, constituyendo la modalidad determinante de su proceso de urbanización reciente.

\section{Introducción}

Los estudios realizados en México sobre la actividad inmobiliaria suelen privilegiar el enfoque centrado en la promoción como categoría y eje explicativo (Schteingart, 1979 y 1983; Legorreta, 1983). De acuerdo con esta visión, el agente particular que actúa independientemente y dispone de capital inicial propio y/o de crédito es un elemento clave de la actividad y en torno a él se articulan las acciones que realizan el rèsto de los agentes inmobiliarios (terratenientes, constructores, financieros, etc.). Christian Topalov es el autor que más ha desarrollado la teoría sobre este tema y ha realizado una investigación sobre el caso concreto de la ciudad de París durante la década de los sesenta (Topalov, 1974, 1975 y 1979). En su estudio acerca de los promotores inmobiliarios, se evidenció que éstos operan con un capital de circulación independiente de los demás capitales implicados en la producción inmobiliaria. Su participación consiste en acelerar el proceso de rotación del capital, al adelantar un capital para la adquisición de la tierra (liberándola para que en ella se pueda construir) y al financiar en parte y/o conseguir financiamiento (generalinente

* Este artículo resume los aspectos centrales de una investigación realizada en el Instituto de Investigaciones Sociales de la UNAM.

** Investigadora del Instituto de Investigaciones Sociales de la UNAM. 
de capital bancario) para que se lleve acabo la construcción. Este promotor se apropia de parte de la ganancia que correspondería al dueño de la tierra e igualmente de una parte que corresponde al constructor (véase Topalov, 1979). La relevancia de la investigación de Topalov es doble: analiza la modalidad de acción inmobiliaria más importante en una ciudad y en un periodo determinado, aportando así un elemento clave para interpretar la intervención de la promoción inmobiliaria en el desarrollo urbano, y explica la razón de la participación de este tipo de capital. Define así la lógica de acción de un nuevo tipo de agente y presenta las posibles combinaciones de esa actividad con las de otros agentes dentro del sistema, válidas para su caso de estudio.

Si bien algunas características muy generales de los promotores pueden encontrarse en nuestro estudio, resulta evidente que la descripción detallada de los mismos no se aplica al caso de Querétaro. El desarrolo del sector inmobiliario, entre 1960 y 1982 , en esa ciudad presenta una serie de peculiaridades que pasamos a analizar a continuación.

A fin de detectar y analizar la lógica de las acciones inmobiliarias en la ciudad de Querétaro, seleccioné del total de desarrollos inmobiliarios realizados en la localidad los que por su importancia (extensión, ubicación) se convirtieron en los más significativos. De acuerdo con este criterio los desarrollos inmobiliarios estudiados son:

a) Parques Residenciales de Querétaro, realizado por Ingenieros Civiles Asociados (ICA).

b) "Jurica" que, en diferentes tiempos, fue propiedad de Torres Landa, Bustamente, Somex y Banamex, ${ }^{1}$ y

c) Los fraccionamientos de "Casas Modernas, S.A."

$\mathrm{El}$ análisis de estos desarrollos inmobiliarios gira en torno a dos hipótesis centrales: que los principales agentes que operaban en la actividad inmobiliaria en Querétaro durante el periodo estudiado realizaban en sus desarrollos inmobiliarios varias actividades (la construcción, la urbanización, etc.), sin reducirse a la promoción propiamente dicha, y que las características de las actividades desarrolladas por estos agentes en los desarrollos inmobiliarios se debieron principalmente a la facilidad para incorporar grandes extensiones de tierra privada a usos urbanos.

Antes de iniciar el análisis, se realizan breves consideraciones sobre el desarrollo industrial y urbano de la ciudad de Querétaro y la forma en que se dio un mercado inmobiliario informal en la ciudad y su diferencia respecto del mercado formal, y se contempla, de manera conjunta, la actividad inmobiliaria que tuvo lugar en la localidad de 1950 a 1960 . El cuerpo central del trabajo aborda el análisis de la lógica que guió la actividad

${ }^{1}$ En el inciso $C_{2}$ se explicitan los periodos y razones del cambio de propiedad de este desarrollo urbano. 
inmobiliaria realizada por los grupos mencionados. Las conclusiones, con las que se cierra el trabajo resumen y explicitan sus aportaciones centrales.

\section{Industrialización, expansión urbana y actividad inmobiliaria en Querétaro}

Desde el periodo colonial y hasta los años cincuenta del presente siglo, la ciudad de Querétaro fue un centro fundamentalmente comercial y administrativo, aunque se efectuó en ella actividad manufacturera, principalmente textil (véase Super, 1983: capítulo V). A partir de la década de los cincuenta se inicia en ella un proceso de industrialización que cobra auge en los sesenta y logra su mayor expresión en los setenta. Algunas de las transformaciones más importantes que este proceso originó, tanto en la base económica de la ciudad como en su población y en la dinámica urbana general, se presentan a continuación.

\section{A. Industrialización}

En 1960 el estado de Querétaro contaba con 743 industrias manufactureras, que ascendieron a 1315 en 1975; es decir, el incremento fue de $77 \%$. Este porcentaje es significativo si se compara con el correspondiente a nivel nacional que fue de $17.2 \%$ en el mismo lapso (datos de los VII y X censos industriales elaborados por la Dirección General de Estadística de la SPP). En este periodo, el número de personas empleadas en la industria manufacturera creció en $\mathbf{5 4 1 . 4 \%}$ en contraste con el aumento nacional de $94.2 \%$. Adicionalmente, el valor de la producción manufacturera a precios corrientes se incrementó $12268 \%$ contra $765 \%$ del nacional, durante el lapso aludido.

Si bien los datos anteriores corresponden a todo el estado, su parte más importante está representada por la ciudad de Querétaro, debido a que en ella y en San Juan del Río se concentró la industria de la entidad. Además, el impacto industrial de San Juan del Río sólo cobró importancia a partir de 1975.

\section{B. Crecimiento poblacional}

Entre 1940 y 1960 la población de la ciudad de Querétaro creció a un ritmo menor que la del resto del país. Sin embargo, de 1960 a 1970 la población se incrementó $66.9 \%$ y de 1970 a $1980,148.9 \% .^{2}$ Estas tasas exceden

${ }^{2}$ Datos de los censos generales de población y vivienda de 1960,1970 y 1980 
a las nacionales y a partir de 1960 , Querétaro, de ser una ciudad de emigración, se convirtió en una localidad de atracción.

\section{Expansión de la mancha urbana}

Como efecto del desarrollo económico y del incremento poblacional, la ciudad de Querétaro acusó un crecimiento importante. La mancha urbana, que en 1950 era de 440 hectáreas, pasó a 3267 en 1980, es decir, creció 1 139\% (más de 11 veces) (véase SAHOP, 1979:158). El efecto combinado de la industrialización y, en particular, de la actividad inmobiliaria produjo un crecimiento especulativo de la mancha urbana.

\section{Mercado inmobiliario informal e inicio del formal}

Hasta donde sé, no existe ningún trabajo que estudie las operaciones de compraventa de tierra y vivienda en la ciudad de Querétaro desde sus orígenes hasta 1950. Por otra parte, en los planos de la ciudad correspondientes a 1867 y 1950 (véase Gobierno del estado de Querétaro, 1978: láminas XXVIII y XXXIV), se advierte que el crecimiento de la ciudad fue muy reducido en dicho lapso. A pesar de que las transacciones de solares rurales y urbanos o viviendas urbanas constituyeron una práctica común, debe enfatizarse que se trataba de acciones aisladas y realizadas directamente entre particulares. Es decir, no existía un agente específico cuya función principal fuera obtener ganancias a través de las operaciones inmobiliarias. En otros términos, aunque había un mercado no organizado de tierra y vivienda, no puede hablarse en sentido estricto de producción ni de promoción inmobiliarias.

Esta precisión no invalida el hecho de que los terratenientes y los comerciantes concentraran la propiedad de solares y viviendas urbanas así como de tierra agrícola próxima a la ciudad. Obviamente de esta concentración de tierra y vivienda se derivaban la obtención y acumulación de rentas tanto agrícolas como urbanas.

La hipótesis de la inexistencia de un mercado formal de tierra y vivienda urbanas hasta la década de los años cincuenta se confirma al comprobarse que hasta 1949 no se registró la creación de ningún fraccionamiento en la ciudad de Querétaro, ni operaciones sistemáticas de compraventa de tierra sin fraccionar, ni venta organizada de casas o lotes urbanos. Este hecho debe ser considerado, puesto que implica una modalidad particular de mercado inmobiliario en la ciudad de Querétaro. El surgi-

elaborador por la Dirección General de Estadística (la Sedue de Querétaro considera estos datos censales inferiores a los reales). 
miento en ella de un mercado inmobiliario formal está vinculado con su desarrollo industrial.

Entre 1950 y 1960 se crearon los siguientes fraccionamientos: "La España", "Niños Héroes", "La Pastora", "Colonia Casa Blanca", "Cimatario", "Jardines de Querétaro" y "Club Campestre". ${ }^{3}$ Un dato común de los siete fraccionamientos es que fueron construidos sobre tierras agrícolas pertenecientes a ex haciendas colindantes a la ciudad. Asimismo fueron desarrollados por propietarios locales de tierra agrícola que no contaban con experiencia previa en la actividad inmobiliaria. Sin embargo, los datos obtenidos indican que no hubo una participación de capitales ni empresas externas a la localidad.

De los fraccionamientos correspondientes al periódo, cuatro - "La España", "La Pastora", "Niños Héroes" y "Colonia Casa Blanca" - son de carácter popular. ${ }^{4}$ Constituyeron prolongaciones cuasi naturales de la extensión de la mancha urbana en el periodo de referencia. De los tres fraccionamientos restantes, dos - "Cimatario" y "Jardines de Querétaro"estaban destinados a las capas medias, mientras que "Club Campestre" se planeó como zona residencial exclusiva. Lo anterior indica que en esta primera fase del desarrollo inmobiliario existió una oferta de lotes o tierra para los sectores de escasos recursos, pero no de vivienda. La información recabada a través de informantes calificados indica que los propietarios de la tierra agrícola se transformaron en agentes inmobiliarios ante las perspectivas que ofrecía el desarrollo industrial de la localidad.

\section{Las grandes acciones inmobiliarias: 1960-1982}

\section{A. La dimensión global de las acciones}

Durante los 22 años que comprende este periodo de las operaciones inmobiliarias, en la ciudad de Querétaro se registra una intensa actividad. Dentro de este lapso pueden distinguirse tres etapas. La primera corresponde a la fase 1964-1967, cuando se iniciaron las intervenciones más importantes, que son las que se desarrollarían en el resto del periodo. La segunda abarca de 1967 a 1973 y en ella surgieron múltiples fraccionamientos impulsados por distintos agentes. En la tercera etapa (1974-1982) pueden distinguirse varias fases: en los años 1974-1975 disminuyó la creación de nuevos fraccionamientos, la cual se recuperó en 1976; este repun-

${ }^{3}$ Estos datos fueron obtenidos de las siguientes fuentes: Obras públicas del estado de Querétaro, Planos catastrales de la ciudad de Querétaro e información proporcionada por el arquitecto Martín Larios.

${ }^{4}$ Los términos "fraccionamiento popular, residencial y campestre" son tomados de la clasificación de la Ley núm. 30 de fraccionamientos del estado expedida en 1961. 
te tuvo un salto importante en 1980, cuando se compraron 13 millones de $\mathbf{m}^{2}$, que representan la adquisición de tierra más espectacular en la historia de la localidad y que provocó un auge de la especulación inmobiliaria. La actividad inmobiliaria decreció a partir de fines de 1982.

El número total de fraccionamientos autorizados en el periodo es de 166 (véase el plano 1), y el área total comprendida por ellos, 21 millones de $\mathrm{m}^{2}$. Los propietarios que vendían (o fraccionaban) la tierra eran terratenientes locales o regionales. Los fraccionadores que adquirían la tierra eran igualmente locales o regionales, excepto en el caso de las adquisiciones realizadas por ICA y Bustamante, a las que se aludirá más abajo.

En cuanto al tipo de fraccionamientos creados, pueden distinguirse tres modalidades: residenciales, campestres y de "clase media". Los fraccionamientos residenciales y cempestres cuentan en general con una adecuada infraestructura; la diferencia entre ambos estriba en la mayor proximidad a la mancha urbana por parte de los residenciales en relación con los campestres, y en el mayor tamaño de los lotes en el caso de los campestres. Los fraccionamientos catalogados como "clase media" estaban destinados a sectores variados que abarcaban desde comerciantes y profesionistas hasta obreros especializados.

\section{B. Crecimiento urbano y tierra ejidal}

Al igual que otros centros urbanos importantes del país, en torno a la ciudad de Querétaro existen varios ejidos. Del total del municipio, $58 \%$ corresponde a tierra ejidal (Gobierno del estado de Querétaro, 1980: 113). La participación de la tierra ejidal en el desarrollo urbano de la ciudad de Querétaro se dio en dos niveles. El primero es la creación de parques industriales; para ello, el Estado expropió el ejido "El Salitre" a fin de crear el parque industrial "Benito Juárez". Asimismo en 1960 ICA, por medio de Parques Industriales, adquirió del Estado 2.25 millones de $\mathrm{m}^{2}$ de tierra de origen ejidal. Es decir, en esta fase la tierra ejidal expropiada tuvo un uso fundamentalmente industrial. La segunda modalidad de uso de tierra ejidal fueron los asentamientos irregulares. Con anterioridad a 1967 predominó el asentamiento relativamente aislado de familias o pequeños grupos de colonos en los ejidos de "Lomas de Casa Blanca", "San Pablo", "Menchaca" y "Bolaños", que se encontraban próximos a la mancha urbana o muy cercanos a ella. Después de la fecha mencionada, se registra la invasión o toma masiva en esos mismos ejidos, destacándose el caso de "Lomas de Casa Blanca".

Los datos anteriores significan que en el periodo considerado la construcción de fraccionamientos habitacionales no tuvo relación con la tierra ejidal. 


\section{La actividad inmobiliaria de los grandes grupos en Querétaro.}

Del total de fraccionamientos construidos en la ciudad de Querétaro de 1960 a 1982 destacan los realizados por tres grupos: ICA, los diferentes propietarios de "Jurica" y "Casas Modernas" (vease el plano 1). Su peso económico y dimensión son diferentes. ICA opera en el ámbito nacional e incluso internacional; los dueños de "Jurica" se han distinguido por su capacidad inmobiliaria y financiera, mientras que "Casas Modernas" es una empresa de carácter estrictamente local. Asimismo, la presencia de estos grupos en la actividad económica del estado, municipio y ciudad de Querétaro es diferente. A reserva de las precisiones que se asientan más adelante, es importante destacar la actividad industrial realizada por ICA en la localidad; también es notable la presencia de Banamex en Querétaro, no sólo como institución financiera sino como accionista de importantes industrias locales y como agente inmobiliario. A diferencia de ICA y de Banamex, "Casas Modernas" se dedica únicamente a la actividad inmobiliaria. A continuación se aportarán elementos generales de identificación de estos grupos, concentrándose el análisis en la actividad inmobiliaria realizada por ellos en la ciudad de Querétaro.

\section{La acción inmobiliaria de ICA}

Creado en 1947, el grupo ICA se ha dedicado desde entonces a las siguientes actividades: construcción pesada, construcción industrial, construccción urbana, estudios técnicos de ingeniería y desarrollo inmobiliario; hacia 1982 era un grupo que contaba con 80 empresas y más de 80000 empleados. Hasta el momento de la nacionalización de la banca (1982) poseía relaciones corporativas para el financiamiento con el Banco del Atlántico. La relación entre este grupo y el Estado mexicano ha sido muy estrecha, y ha estado personificada en gran parte en la figura de Bernardo Quintana, además de otros funcionarios de alto nivel del Estado que han sido miembros del cuerpo directivo del grupo (véase Fragoso, Concheiro y Gutiérrez, 1979: 142).

En el ámbito urbano y regional, la actividad que realiza este grupo es amplia y diversificada. Esta injerencia lo convierte en el más importantes monopolio de la construcción. Por el tipo de obras, su cliente principal es el Estado, por medio de sus secretarías y empresas públicas (Comunicaciones y Transportes, Agricultura y Recursos Hidráulicos, Pemex, DDF, CFE, etcétera).

En cuanto a la acción inmobiliaria de ICA en Querétaro destaca la adquisición v urbar ización de tierra. La compra de tierra por parte de ICA presenta dos cara terísticas importantes. La primera es la situación agrícola de la tierra aı quirida tanto a particulares como al propio Estado (eji- 


\section{PLANO 1}

\begin{tabular}{|c|c|}
\hline 1 Villas del Mesón & 41 Corregidora de Querétaro \\
\hline 2 Jurica & 42 Prados del Mirador \\
\hline 3 Raquet Club & 43 Vista Alegre \\
\hline 4 Huertas la Joya & 44 Balaustrados \\
\hline 5 Colinas del Bosque & 45 Observatorio \\
\hline 6 Club Campestre & 46 Valle Alameda \\
\hline $\begin{array}{l}7 \text { Álamos 1a. sec. } \\
8 \text { Álamos 2a. sec. } \\
9 \text { Álamos sec. Jardín }\end{array}$ & 47 Mercurio \\
\hline 10 Balcones & 51 Santa Bárbara \\
\hline 11 Colinas del Parque & 52 Peñuelos \\
\hline 12 Arboledas & 53 Cd. Satélite \\
\hline 13 Loma Dorada & 54 El Carambullo \\
\hline 14 Vista Hermosa & 55 Insurgentes \\
\hline 15 Zona Dorada & 56 El Sol \\
\hline 16 Constituyentes & 57 Col.Obrera \\
\hline 17 San Javier & 58 Industrial \\
\hline 18 Bosques del Acueducto & 59 Progreso \\
\hline 19 Calesa 2a. sec. & 60 U. H. Arteaga \\
\hline 20 Calesa 1a. sec. & 61 El Porvenir \\
\hline 21 Jardines de Querétaro & 62 España \\
\hline 22 Carretas & 63 Los Molinos \\
\hline 23 Quintas de Marqués & 64 Los Alcanfores \\
\hline 24 Colinas del Cimatario & 65 Los fresnos \\
\hline 25 Cimatario & 66 La Florida \\
\hline 26 Villas del Sur & 67 Constitución 1917 \\
\hline 27 Lomas de Querétaro & 68 La Era \\
\hline 28 Jardines de la Hacienda & 69 Las Rosas \\
\hline 29 El Jacal & 70 Constituyentes 1824 \\
\hline 30 Las Plazas & 71 San Antonio \\
\hline 31 Mansiones del Valle & 72 Las Hadas \\
\hline 32 Colonia del Valle & 73 Las Palmas \\
\hline 33 Residencial Tejeda & 74 El Marqués \\
\hline 34 Santiago & 75 Villas del Sol \\
\hline 35 Niños Héroes & 76 Aragón \\
\hline 36 Villas de las Arboledas & 77 Paseo de los Arcos \\
\hline 37 Ensueño & \\
\hline 38 Virreyes & \\
\hline 29 Estrella & \\
\hline 4) Misio tes & \\
\hline
\end{tabular}

\section{COLS. POPULARES}

101 Emiliano Zapata 102 San José los Olvera 103 Reforma Agraria 104 Lomas de Casablanca 105 Casablanca 106 Bolaños 107 Menchaca 108 Las Américas 109 Loma Bonita

\section{ZONAS INDUSTRIALES}

A 1950

B Parques ind ustriales 1966

C Parque "San Pedrito Peñuelos"'

D Parque "Benito Juárez"

$$
1972
$$

E 1979

F Fertimex

G Parque "Bananera"

$\mathrm{H}$ Purina

J 1980

$\mathrm{K}$ Levis

\section{EQUIPAMENTO}

201 Cerro de las campanas. C.U.

202 Alameda 203 IMSS

204 Campo Militar

205 Auditorio

206 Unidad Deportiva

Fuentes: Larios G., Martín: plano IV. Área urbana de la ciudad de Querétaro. 
dos expropiados); es decir, ICA no compró predios urbanos de ningún tipo (centrales o periféricos, pequeños o grandes). La segunda característica es el volumen de las adquisiciones de tierra realizadas por el grupo. Considerando tanto la tierra ocupada por fraccionamientos industriales y residenciales como la que es detentada en reserva por la empresa, su área asciende a 6.5 millones de $\mathrm{m}^{2}$. Esta cifra representaba $39 \%$ de los 16.6 millones de $\mathrm{m}^{2}$ del área comprendida en el perímetro urbano de la ciudad de Querétaro en 1951, según el Diario Oficial del gobierno del estado. Exceptuando la tierra de origen ejidal, la mayor parte de la restante correspondía a antiguas haciendas.

El convenio fiscal para la adquisición de la tierra fue realizado por "Parques Industriales de Querétaro" a nombre de ICA y las operaciones inmobiliarias fueron realizadas por una de las empresas que componen el grupo "Administración Inmobiliaria, S.A." (AISA), que nació en Querétaro, y que maneja sus desarrollos inmobiliarios. De lo anterior se deduce que ICA, por medio de AISA y de otras divisiones del grupo, actuó como comprador, proyectista, urbanizador, constructor y administrador de los fraccionamientos. Por lo que se refiere al financiamiento de las operaciones, el capital provino del Banco Internacional Inmobiliario, S.A., que después se convirtió en el Banco del Atlántico.

En relación con la compra de tierra, destaca su adquisición masiva en puntos estratégicos de la ciudad de acuerdo con el desarrollo urbano previsto a corto y mediano plazos. Aunque no se puede establecer una relación de causalidad entre la construccción de la vía rápida MéxicoQuerétaro y la adquisición de tierra por parte de ICA en la localidad, ambos hechos fueron próximos en el tiempo. Al conocimiento que el grupo poseía sobre el desarrollo económico y urbano del país, se sumó, en este caso, la apreciación concreta de la potencialidad del mercado inmobiliario en la ciudad de Querétaro. Este factor, aunado a su poder económico y político, facilitó la compra de tierra en grandes cantidades. La disponibilidad de tierra agrícola concentrada en un número relativamente reducido de propietarios contribuyó sin duda a esta adquisición.

Con respecto a la elección del tipo de fraccionamiento, debe destacarse la estrategia inicial de ICA según la cual los fraccionamientos se diferenciaban en industriales, campestres, residenciales y populares con el fin de diversificar la oferta y captar todo el mercado potencial. Esta propuesta fue formulada en 1963, al solicitarse las autorizaciones de fraccionamientos. Sin embargo, el primer fraccionamiento residencial realizado por ICA en Querétaro fue registrado en 1966. Entre ambas fechas mediaron dos circunstancias: la evaluación por parte de ICA de la experiencia de los fraccionamientos campestres, tanto en Querétaro como en el resto del país. La venta relativamente lenta de los lotes campestres y la construcción mínima de vivienda sobre los mismos retardaba la consolidación de este tipo de fraccionamientos. Por otra parte, para ICA el margen de 
ganancia en un fraccionamiento urbano residencial era comparativamente mayor (debido, entre otras cosas, al tamaño de los lotes y a los precios correspondientes), sobre todo considerando la ubicación respecto de la ciudad de la tierra adquirida por ellos. Estas razones apoyaban la supresión de los fraccionamientos campestres en su proyecto inmobiliario en Querétaro. En lo que respecta a la propuesta de fraccionamiento popular, destaca el área inicial reservada a él (506 $967 \mathrm{~m}^{2}$ ), así como el hecho de que esta cantidad era incluso mayor que la planeada para fraccionamientos residenciales ( $421182 \mathrm{~m}^{2}$ ); sin embargo, a esta última cifra habría que agregar la correspondiente a los fraccionamientos campestres (2 041000 $\mathrm{m}^{2}$ ), con el objeto de poder comparar el proyecto de oferta de tierra de ICA para los sectores mayoritarios en relación con las clases restantes. A pesar de ello, llama la atención que ICA haya planeado ofrecer los fraccionamientos populares pensando no sólo en los trabajadores de sus empresas en la localided sino también en los de las zonas industriales de Querétaro como potenciales adquirientes de tierra. El hecho de que no se creara ningún fraccionamiento popular ${ }^{5}$ está relacionado con dos factores de distinta índole. El primero, de tipo económico, se vincula con la estrategia según la cual la apertura de nuevos fraccionamientos está supeditada al avance de las ventas de los lanzados con anterioridad y a la detección de una probable nueva demanda. El segundo se refiere a la ubicación de la fuerza de trabajo en el municipio; ésta se localiza fundamentalmente fuera de la mancha urbana y en particular en tierra ejidal, circunstancia que "justificaba" postergar la creación de fraccionamientos populares.

Descartados los fraccionamientos de tipo campestre y popular, quedaban únicamente los residenciales como variante a través de la cual ICA realizaría en Querétaro la actividad inmobiliaria. La tierra urbanizada a la fecha fue lotificada y vendida en distintos periodos acordes con la consolidación de las ventas en los fraccionamientos previos y con la detección de una demanda solvente (véase el cuadro 1). Ciertamente, al seleccionar la ubicación de sus nuevos fraccionamientos, ICA consideraba el avance de la urbanización en la localidad, el cual estaba, en parte, condicionado por la consolidación de sus propios desarrollos inmobiliarios.

En los fraccionamientos residenciales, ICA tampoco construyó un gran número de viviendas, lo que hace evidente que su objeto fundamental era impulsar el avance de las ventas de lotes; es decir, que el eje de su actividad inmobiliaria era la venta de tierra urbanizada. Parecería incongruente que el mayor consorcio de la industria de la construcción no edificara un número importante de viviendas en la localidad. La explicación se encuentra en el menor margen de ganancia que ICA parece obtener de la pro-

${ }^{5}$ Aunque sí construyó un número reducido de viviendas (28) para trabajadores de sus empresas. 
ACCIONES INMOBILIARIAS EN QUERÉTARO

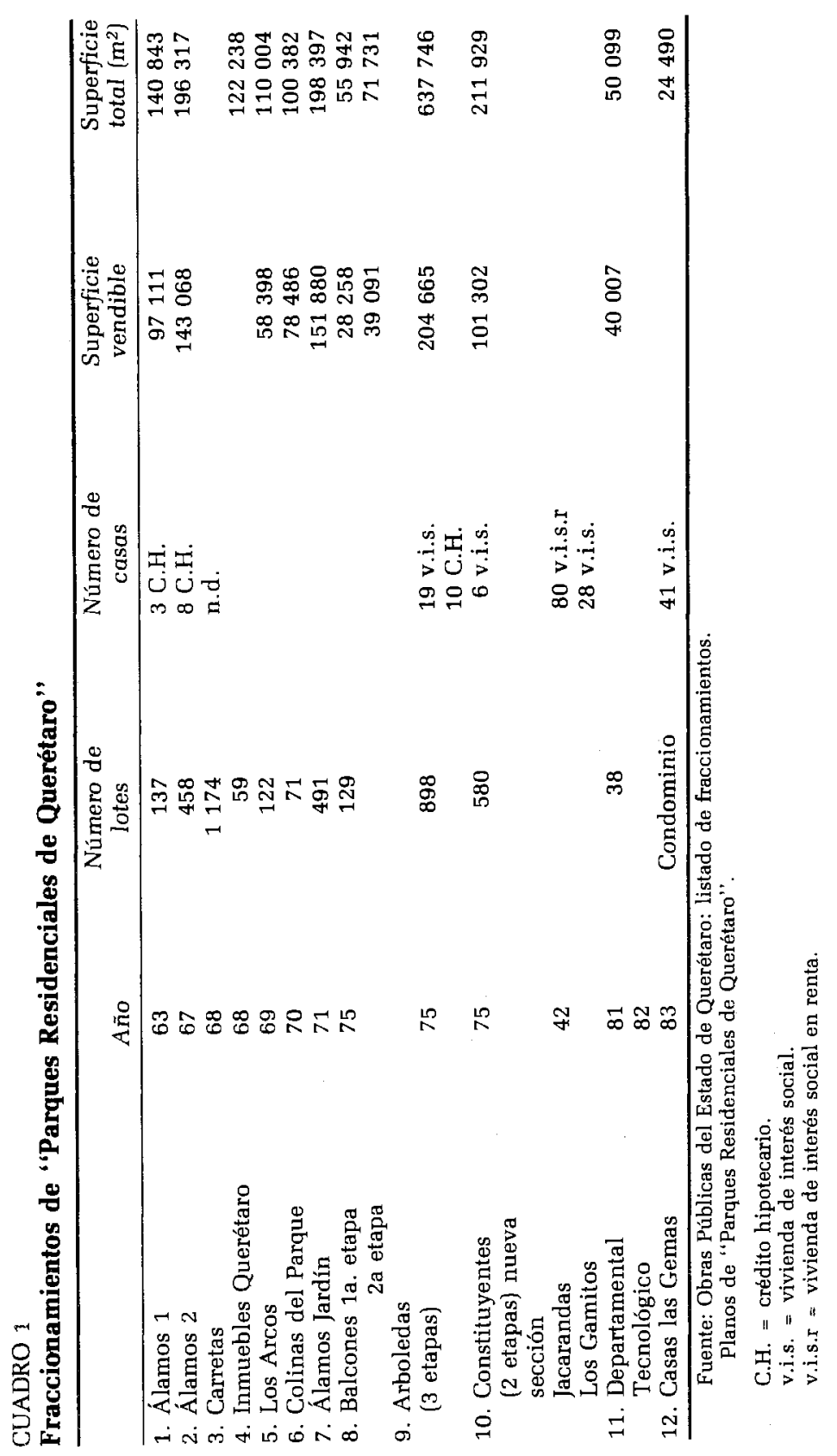


ducción habitacional comparado con la construcción civil u obra pesada, o con la propia venta de tierra. Esta ganancia menor es causada por la baja escala en que debía edificarse en Querétaro en relación con la capacidad técnica y operativa del consorcio; asimismo, el tamaño reducido de la demanda solvente en la localidad desalentaba, de antemano, la construcción de un importante número de viviendas residenciales.

En el manejo de los fraccionamientos residenciales por parte de ICA, la totalidad de las fases que incluye la acción inmobiliaria, así como de los agentes que intervienen en ella y de sus ganancias respectivas, están en el interior del grupo. En otros términos, ello significa que (excepto para la obtención de la tierra rural), el grupo y sus acciones inmobiliarias no dependen de ningún otro factor externo; se trata, en consecuencia, de una actividad con una lógica monopólica que le permite actuar con notable ventaja respecto de los fraccionadores competitivos y, sobre todo, establecer un precio monopólico en la venta de los lotes urbanizados. En la medida en que se eliminan la mayoría de los impedimentos de la acción inmobiliaria competitiva, el precio ${ }^{6}$ puede garantizar un alto margen de ganancia, aun en el caso de que se determinen precios similares a los del mercado inmobiliario local.

El impacto urbano que la intervención de ICA tuvo en Querétaro se efectuó en varios niveles: 1) en la realización de infraestructura vial (construcción de la vía rápida México-Querétaro), 2) en el impulso al desarrollo industrial de la localidad (creando parques industriales e instalando sus propias industrias en ellos), y 3) en la actividad inmobiliaria relacionada tanto con el desarrollo industrial como habitacional.

Con respecto al balance de conjunto de este impacto, es evidente que la vía rápida México-Querétaro incrementó los nexos entre estos dos puntos clave del territorio nacional. Por su parte, los fraccionamientos industriales facilitaron el desarrollo de las actividades secundarias en una ciudad tradicionalmente administrativa y comercial; las propias industrias que ICA estableció en la localidad afianzaron este desarrollo. Por último, los fraccionamientos habitacionales de ICA apoyaron el desarrollo de la ciudad, tanto por su ubicación como por la concepción urbana de los mismos. Sin embargo, a pesar de la planeación de las acciones de ICA, éstas indujeron un crecimiento artificial de la ciudad e impulsaron la especulación inmobiliaria.

\section{2. "Jurica" o la tierra como eje de la ganacia inmobiliaria}

El fraccionamiento "Jurica" se encuentra ubicado en el noroeste de la ciudad de Querétaro; en el momento de su creación (1968) se hallaba fuera trabajo.

${ }^{6}$ El análisis específico de los precios de los terrenos no es objeto de este 
de la mancha urbana, pero actualmente se encuentra unido a ella. Este desarrollo inmobiliario se realizó en una propiedad rural de 3 millones de $\mathrm{m}^{2}$ (que reunía condiciones óptimas para la producción agrícola tanto por la calidad de la tierra como por la facilidad de riego), y que formaba parte de un área mayor. Quizá por estas razones esta propiedad agrícola tenía un decreto de inafectabilidad. Sin embargo, los campesinos de la zona iban a solicitar la ampliación de ejidos con base en ella. Para evitar esta afectación, el terratenientes utilizó un recurso hábil: vender la tierra para que se convirtiera en un fracccionamiento campestre. El vendedor de la tierra pertenecía a uno de los grupos terratenientes de Querétaro (los Urquiza) y poseía otras propiedades en la entidad.

En la historia de este fraccionamiento deben distinguirse cuatro etapas bien definidas. En la primera fase (1968 a 1974) se realizó el cambio de uso del suelo (de rural a urbano) y se urbanizó la mayor parte de los 3 millones de $\mathrm{m}^{2}$; para ello, se pidieron dos permisos de fraccionamientos. ${ }^{7}$

El proyecto de fraccionamiento de la zona fue realizado por la Inmobiliaria y Comercial Bustamante (Incobusa) y las obras de urbanización por Ocimex, que era la compañía urbanizadora de este grupo. La urbanización se caracterizó por lotes con un promedio de $2500 \mathrm{~m}^{2}$, extensión relativamente normal para un fraccionamiento campestre.

Aproximadamente $60 \%$ de la operación fue realizada con un crédito de la Financiera Intercontinental. Otro dato relevante es que en el proyecto inicial se planeaba vender únicamente lotes urbanizados, ya que esta estrategia permitía disminuir el monto de la inversión y una rotación más rápida del capital. El plan inicial de vender sólo lotes no se mantuvo totalmente, pues el fraccionador edificó 16 casas de $200 \mathrm{~m}^{2}$ de construcción para "calentar el negocio", es decir, para impulsar las ventas de los lotes. La ganacia calculada en la operación $(100 \%)$ fue relativamente alta.

Durante este periodo, el total de casas construidas por los compradores de los predios fue de 80 . Esta cifra parece baja y, entre otras cosas, hace suponer que la compra de lotes no se realizaba primordialmente para que el adquiriente construyera una vivienda para descanso, sino con una intención especulativa en mayor o menor grado.

En 1974 Torres Landa vendió el fraccionamiento a Alberto Bustamante (hijo del principal accionista de Incobusa), iniciándose una segunda etapa que fue la más importante (1974 a 1978). Durante ella, destacan dos hechos: el cambio de patrón de lotificación y la adquisición de nuevas propiedades colindantes con el fraccionamiento, como parte de un pro-

${ }^{7}$ El comprador fue Juan José Torres Landa, quien en el momento de la operación era gobernador del estado de Guanajuato y había sido subdirector adjunto de uno de los grupos inmobiliarios más importantes del país en la década de los setenta, la Inmobiliaria y Comercial Bustamante (Incobusa). 
yecto de grandes alcances. En relación con el primer aspecto, Bustamente urbanizó la tierra en breña adquirida con base en lotes de $1000 \mathrm{~m}^{2}$ y de $525 \mathrm{~m}^{2}$, con un precio de venta en 1974 que oscilaba entre 150 y 200 pesos por $\mathrm{m}^{2}$ según la ubicación y el tamaño. Bustamente adquirió extensiones importantes de tierra colindantes con "Jurica", que junto con la anterior sumaban 13 millones $\mathrm{de}^{2}$. Las nuevas propiedades adquiridas se conocen con los nombres de "Mesón del Prado", "Juriquilla" y "San Isidro"'. ${ }^{8}$ Sin embargo la ampliación desmedida de las adquisiciones de tierra por parte de Alberto Bustamante en diferentes ciudades del país provocaron dificultades financieras para su empresa, lo cual afectó a "Jurica". Por tal razón, la totalidad del proyecto inmobiliario (13 millones de $\mathrm{m}^{2}$ de tierra más las instalaciones que incluía) pasó al Fideicomiso Liquidador de la Secretaría de Hacienda y Crédito Público; con éste surge la tercera etape de "Jurica" (1978-1979). Durante ella, no se urbanizaron nuevas áreas y, por ello, no se realizó ninguna venta de lotes. Esta actitud se explica porque la finalidad del Fideicomiso era vender la propiedad para recuperar lo más rapidamente posible una parte del adeudo de Bustamante. En enero de 1980, Banamex adquirió el fraccionamiento "Jurica" en 1275 millones de pesos. ${ }^{9}$ Los aspectos relevantes de esta última etapa de "Jurica" son tres: el cambio de la concepción del fraccionamiento, la ampliación de las actividades inmobiliarias de Banamex y el proyecto de desconcentración de la casa matriz de Banamex a "Jurica".

Banamex pretendió dar un giro nuevo al fraccionamiento convirtiéndolo de campestre en una zona típicamente urbana y comercial. Este plan se realizó en la parte de "Jurica" que quedó sin lotificar desde que era propiedad de Torres Landa. El cambio consistió en poner a la venta lotes de $200 \mathrm{~m}^{2}$ en promedio, y esta misma concepción se pensaba aplicar a las zonas no urbanizadas de "Juriquilla" y "Mesón del Prado". Esta parte del proyecto, así como la realización de zonas comerciales, quedaron detenidas por la nacionalización de la banca y sus activos, que incluyó esta propiedad de Banamex.

La modificación de los proyectos urbanísticos anteriores por parte de Banamex estuvo acompañada de la creación de un complejo aparato administrativo, urbanizador y constructor; destacaba la creación de la inmobiliaria UMBRA, de la cual Banamex detentaba $99 \%$ de las acciones, según informó la Subsecretaría de la Banca al poner a la venta las acciones de la banca privada nacionalizada. Este hecho indica por sí solo la importancia financiera que para Banamex tenía "Jurica" que, en parte, estaba relacionado con el proyecto de reubicación o desconcentración de

${ }^{8}$ No obstante la nueva y masiva adquisición de tierra, el fraccionamiento en su conjunto sigue llamándose "Jurica" y la tierra anexada al proyecto original como "Jurica segunda sección" (ver Plan de Desarrollo Urbano de Querétaro, 1982, plan director urbano: 6 y 7 ).

${ }^{9}$ Según informe oficial de Banamex, fechado el 26 de marzo de 1980 
las oficinas centrales de Banamex a "Jurica". Para ello, el grupo puso en marcha todo un plan, que fue frenado por la nacionalización.

Según informes de un ejecutivo de Banamex en Querétaro, a finales de 1984 este grupo bancario nacionalizado mantenía la propiedad de 1.5 millones de $\mathrm{m}^{2}$ en función de la reubicación institucional.

$\mathrm{Al}$ sintetizar la experiencia del fraccionamiento "Jurica", deben enfatizarse varios hechos. El primero, el más impactante, es el volumen de la tierra adquirida ( 13 millones de $\mathrm{m}^{2}$ ). Por una parte, esta compraventa está relacionada con un número reducido de propietarios agrícolas que facilitaron su adquisición y, por otra, con tres adquirientes (Torres Landa, Bustamante y Banamex) dotados de una gran capacidad de compra. Estas condiciones no se reúnen con frecuencia en las operaciones inmobiliarias. Debido a estas circunstancias, el eje de la ganancia en este fraccionamiento pudo ser casi exclusivamente la tierra, puesto que el vendedor concentraba las ganancias del cambio de uso del suelo (de rural a urbano) y la exclusividad en la venta de la totalidad de los lotes. Ambos factores permitieron a los dos primeros dueños (Torres Landa y Bustamante) ofrecer incluso los terrenos a precios no elevados en el contexto del mercado local.

La creación de este fraccionamiento reúne condiciones excepcionales: tamaño, carácter oficial del comprador (gobernador), facilidades para su autorización, acceso al crédito bancario, etc. Sin embargo, parecen haberse cubierto los requisitos legales estipulados para este tipo de fraccionamientos. No obstante, sorprenden varios aspectos; el primero se refiere a la falta de previsión o planeación urbanas en cuanto a las consecuencias que propiciaría la puesta en operación del fraccionamiento. El área total de "Jurica" lo convierte en el fraccionamiento de mayores dimensiones de la localidad. Si se relaciona con el tamaño de la mancha urbana de la ciudad en $1982,{ }^{10}$ equivale a $26 \%$ de la misma. Aunque las instalaciones exigidas para el fraccionamiento fueron financiadas por el fraccionador (redes de agua, luz, etc.) e incluso actualmente él está costeando los gastos de recolección de basura, mantenimiento de áreas jardinadas y vigilancia, lo importante de la repercusión urbana y financiera de estos servicios no son sólo sus instalaciones, sino la operación del servicio mismo. Un ejemplo claro es el del agua; al margen de las redes correspondientes, lo importante es la existencia y dotación suficiente del líquido. Al respecto, el consumo de agua de un fraccionamiento campestre es alto, en particular en lo que se refiere a las áreas verdes y jardinadas de cada lote. Aunque el fraccionamiento cuenta con varios pozos, las reservas corres-

${ }^{10}$ Según el Plan de Desarrollo Urbano de Querétaro, 1982 (5 013 hectáreas). Debe advertirse que esta área corresponde a 1982 y que cuando se iniciaron las ventas en 1968 el tamaño de la mancha urbana era mucho menor. En 1951 era equivalente a 16.69 millones de $\mathrm{m}^{2}$. 
pondientes a éstos no pueden desvincularse de las requeridas para la ciudad en su conjunto. En este punto, se advierten ya serias deficiencias en la ciudad, como se reconoce en los informes de gobierno. ${ }^{11}$ Idéntica argumentación puede establecerse para otros servicios: de transporte, de limpia, de educación, áreas comerciales, etcétera.

Otro elemento importante en la planeación urbana de cualquier ciudad es prever la orientación de su crecimiento futuro de acuerdo con las condiciones naturales del medio, el régimen de propiedad del suelo y usos del mismo (industrial, agrícola, etc). A este respecto, como puede observarse en el plano, el fraccionamiento "Jurica" constituye una prologación artificial de la ciudad y condicionará la expansión de ésta a corto y mediano plazos; en otros términos, dificultará el desarrollo de otras áreas más convenientes de la ciudad (véase Plan de Desarrollo Urbano de Querétaro, 1982). Las consecuencias urbanas de este fenómeno no son imprevisibles.

Como nota final conviene advertir que desde el momento de la nacionalización de la banca privada existe indefinición (o por lo menos falta de información oficial precisa) respecto al propietario actual del fraccionamiento y del futuro inmediato de este desarrollo inmobiliario. Las diferentes fuentes consultadas no han permitido dilucidar este asunto. ${ }^{12}$

\section{3. "Casas Modernas" o la utilización de la lógica}

de los grandes grupos por parte de una inmobiliaria local

La inmobiliaria "Casas Modernas de Querétaro, S.A." demuestra cómo la presencia de grandes grupos en la actividad inmobiliaria induce en este sector de la economía prácticas que obedecen al esquema bajo el cual operan los grandes grupos económicos. Es decir, algunas empresas cuya estructura interna (capital, diversificación, nexos con otras ramas de la economía, peso político, etc.) no corresponde a la de un monopolio, incorporan sus formas y tácticas de operación para poder actuar con ventaja respecto de las restantes empresas del ramo.

"Casas Modernas" se constituyó en la década de los sesenta e inició sus operaciones en 1967. Despues de "Parques Residenciales" y "Jurica”, es la empresa inmobiliaria cuya acción en el periodo considerado es la mayor de entre las 38 que operaban en la localidad. La magnitud de su actividad (20 fraccionamientos) contrasta con el tamaño reducido de su estructura operativa.

${ }^{11}$ Véase quinto informe de R. Camacho G., 25 de julio de 1984. En él se asienta que: "nuestro gasto [de agua] es de $950 \mathrm{l} / \mathrm{seg}$; requerimos de $1450 \mathrm{l} / \mathrm{seg}$ " (p. 28).

${ }_{12}$ Algunos informantes calificados indicaron que los señores Ballesteros, Brenner y Cosío han adquirido parte de las acciones del fraccionamiento. Estos datos no pudieron ser corroborados a través de documentos escritos. 
Con poco personal maneja tanto la inmobiliaria como una casa de materiales para la construcción y una constructora. Sus trabajadores eventuales son contratados tanto para la obra de construcción como para el diseño urbano y arquitectónico, y para la supervisión de obra.

"Casas Modernas" cubre la totalidad de las fases y operaciones que incluye la actividad inmobiliaria; es decir, adquiere la tierra, realiza el diseño urbano y arquitectónico, urbaniza los fraccionamientos, construye viviendas y lleva a cabo la venta de los lotes y de las casas. Para el financiamiento de su actividad utiliza recursos propios; en el caso particular de la construcción de las viviendas, aporta $25 \%$ del monto global y obtiene créditos hipotecarios para el porcentaje restante. Cumple los requisitos reglamentarios y legales estipulados para la actividad inmobiliaria y constructiva en los mínimos tolerados.

Sus 19 fraccionamientos ${ }^{13}$ hasta 1980 han sido construidos sobre tierra urbana. La ubicación de éstos en el interior de la ciudad es diversificada; en general la empresa trata de conseguir terrenos cuya localización próxima a la vialidad, infraestructura y servicios urbanos reduzca los costos de inversión; por ello, aunque no poseen una ubicación céntrica, se encuentran en el interior de la mancha urbana. El área de los fraccionamientos, así como el número de lotes, es variable; la superficie de los lotes fluctúa entre $125 \mathrm{~m}^{2}$ y $200 \mathrm{~m}^{2}$.

Entre los fraccionamientos de "Casas Modernas" predominan los destinados a las capas medias. Éstos poseen los servicios mínimos requeridos. La mayoría de los lotes han sido ya vendidos y el número de viviendas construidas es mucho más alto que en el caso de "Parques Residenciales" y "Jurica" en relación con el número de lotes; en total las viviendas ascienden a 2500 . Las casas son construidas por la propia empresa y, en su mayor parte, han sido financiadas con "crédito para vivienda de interés social" (FOVI-FOGA); son fácilmente vendibles en el mercado. Los datos generales de los fraccionamientos se concentran en el cuadro 2.

Considerando globalmente la actividad de "Casas Modernas", se advierten algunas diferencias en comparación con "Parques Residenciales" y "Jurica". La primera tiene relación con la tierra; todos los fraccionamientos han sido construidos sobre tierra urbana; es decir la empresa no se apropió de las rentas provenientes del cambio de uso del suelo (de agrícola a urbano) y, en consecuencia, debió pagar un precio más alto que ICA o los propietarios de "Jurica". Como ya se señaló, "Casas Modernas" trató de contrarrestar este efecto detectando predios y propiedades en los que fuera posible "reducir" su precio urbano. Es también notable que, disponiendo de una menor capacidad operativa que "Parques Residenciales" y "Jurica", haya construido un número mucho más elevado de

\footnotetext{
${ }^{13} \mathrm{El}$ socio capitalista ha construido además un fraccionamiento campestre cuya extensión es de 5.6 millones de $\mathrm{m}^{2}$.
} 


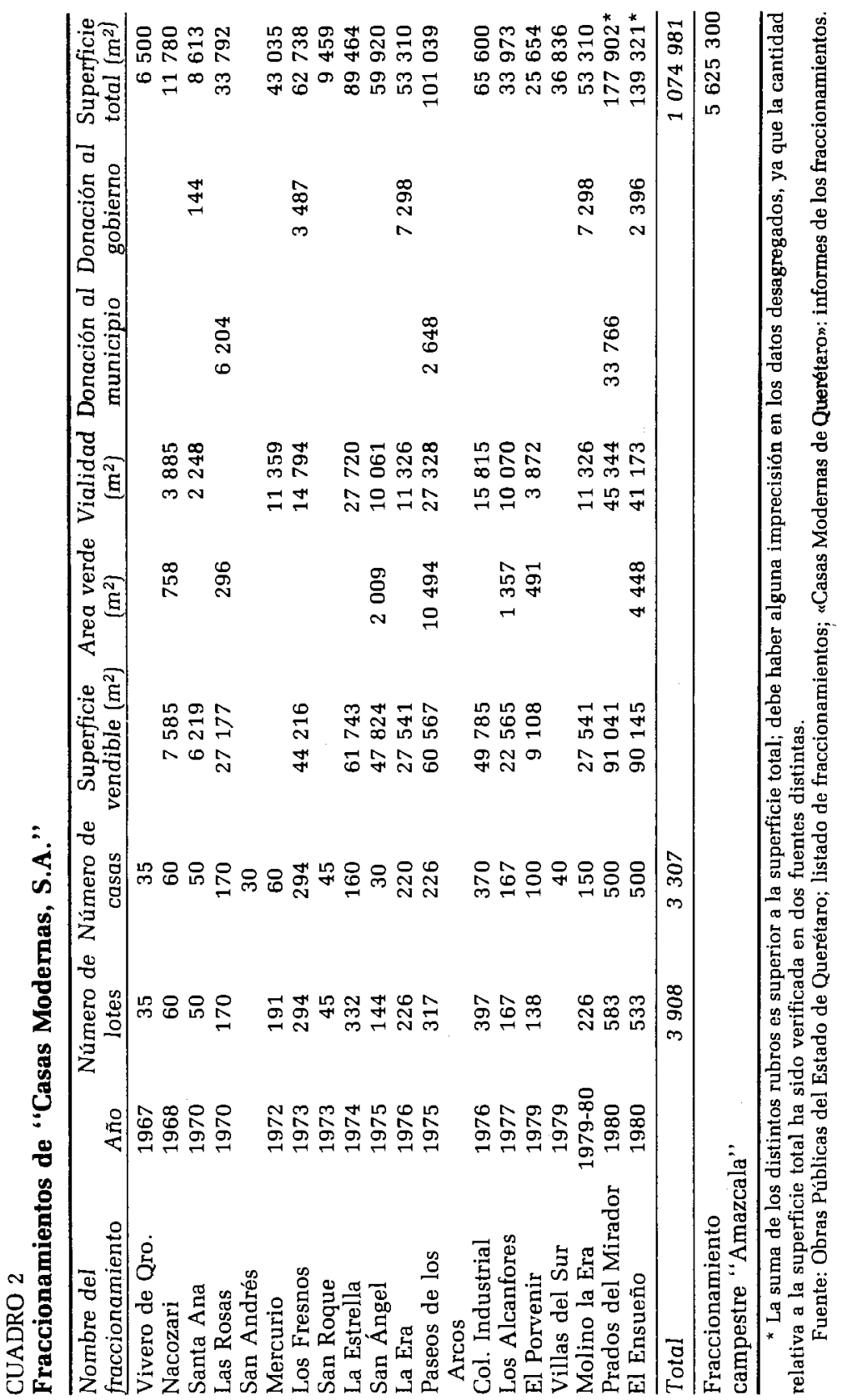


casas, en términos absolutos y relativos. Esto indica que el margen de ganancia para esta empresa proviene, en mayor grado, de la construcción y no de la venta de lotes. Por otra parte, ya que no interviene en su operación ningún agente externo a la empresa, elimina los gastos de intermediación y concentra las ganancias correspondientes. Además, la rotación de su capital es rápida debido a que son sujetos de crédito y a que la venta de lotes y viviendas se ha realizado en lapsos cortos. Por todo lo anterior su ganancia global es alta.

En cuanto al efecto urbano de su actividad, debe señalarse a su favor el hecho de que propicia la consolidación de las zonas en las que establecen sus fraccionamientos. Sin embargo, ya que cubren únicamente los requisitos mínimos de la reglamentación urbana, sus fraccionamientos no contribuyen al mejoramiento de la imagen y calidad de la vida urbana. Para apreciar de manera conjunta las coincidencias y las diferencias en la forma de operar de los grupos inmobiliarios y de las acciones desarrolladas por ellos, se concentran en el cuadro 3 las características más relevantes de sus operaciones.

\section{Conclusión}

Como en otras localidades del país, el crecimiento urbano y el impulso a la actividad inmobiliaria en la ciudad de Querétaro se hallan relacionados con su desarrollo industrial. Así, el inicio del mercado formal inmobiliario en los años cincuenta coincide con el establecimiento de la primera zona industrial en la capital del estado. Posteriormente, en la década de los sesenta, la presencia de ICA incluye el desarrollo de proyectos inmobiliarios e industriales, y en lo que se refiere al desarrollo inmobiliario "Jurica", en la etapa en que fue propiedad de Bustamante, se efectúa casi simultáneamente al establecimiento del parque industrial Benito Juárez.

Respecto a la primera de las hipótesis formuladas al inicio de este ensayo, debe enfatizarse que los grupos inmobiliarios estudiados concentraban y realizaban la totalidad de las actividades que implica el proceso de urbanización y circulación de tierra así como de la producción de vivienda; es decir, no se dio en ninguno de los desarrollos urbanos impulsados por ellos la presencia del promotor inmobiliario en sentido estricto, el que opera de manera au tónoma y articula al resto de los agentes inmobiliarios. Esto no implica anular la función que realiza el promotor, sino que ésta es llevada a cabo por el propio grupo, de tal manera que viene a ser una de las varias funciones desarrolladas por él.

En relación con la segunda de las hipótesis debe resaltarse que en dos łe los grupos estudiados es notoria la cantidad de tierra agrícola privada ıdquirida para sus desarrollos, de la cual detentan todavía importantes reservas para futuras operaciones. Ello fue posible a causa de la capaci- 


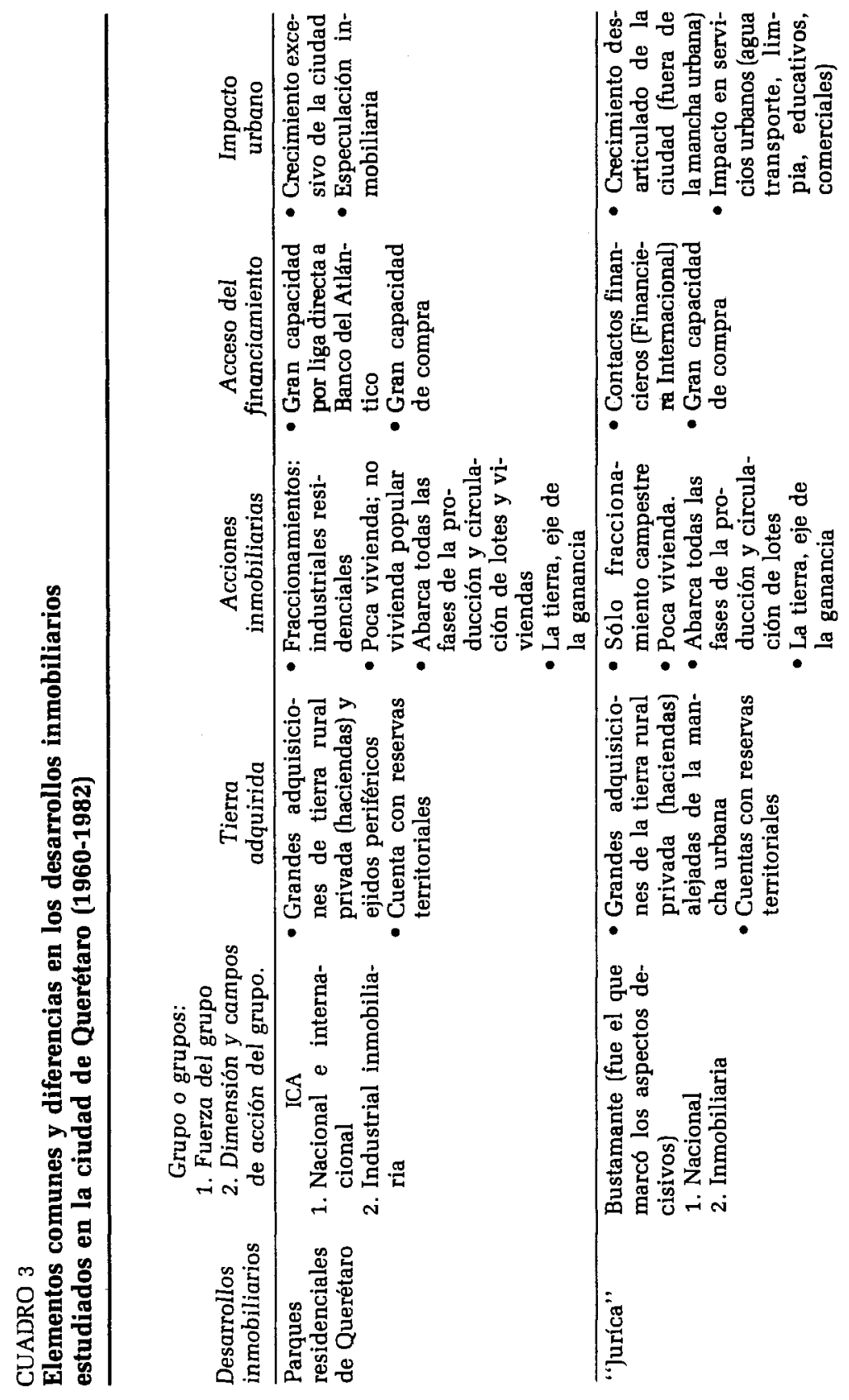



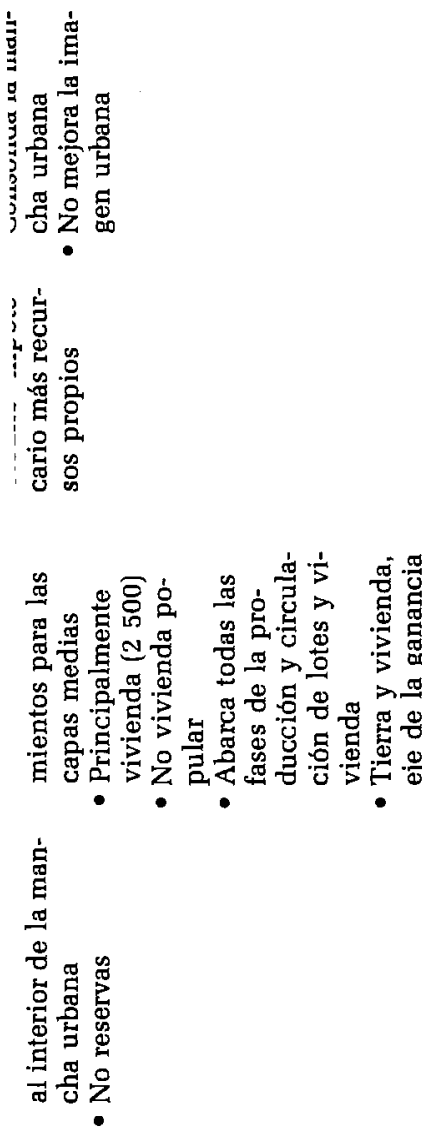

음

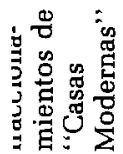


dad económica y facilidad de acceso al crédito por parte de los dos grupos implicados. De este modo concentraron las ganancias del cambio de uso del suelo (de rural a urbano), así como las provenientes de la venta de los lotes urbanizados. Ello les permitió convertir la tierra en eje de la ganancia, e implantar precios cuasi monopólicos en el mercado de tierra urbana. La comercialización de la vivienda fue utilizada por ellos simplemente como un factor de dinamización de la venta de lotes.

Con respecto a ambas hipótesis, es importante subrayar cómo una empresa local que no reunía las características de poder económico y facilidad de acceso al crédito financiero de los grupos más grandes, incorporó la lógica de operación de los dos grupos citados para disponer de un margen de maniobra frente a ellos y poder competir con ventaja frente a las compañías inmobiliarias locales.

En cuanto al impacto urbano de estas operaciones inmobiliarias debe enfatizarse que produjeron una expansión urbana superior a la requerida por el grado de desarrollo industrial y el incremento de la población en la localidad; se trata, por tanto, de un importante crecimiento especulativo de la mancha urbana y del que son causantes, en buena parte, los agentes inmobiliarios. Es altamente significativo que la suma de la tierra adquirida por estos tres grupos (incluyendo las reservas territoriales) asciende a 22.5 millones de $\mathrm{m}^{2}$, lo cual equivale a $45 \%$ del área de la mancha urbana en la fecha de adquisición. El efecto urbano de esta expansión de la actividad inmobiliaria es innegable y fundamentalmente se expresa en el condicionamiento de la futura expansión de la ciudad hacia las reservas de tierra que detentan y, en menor medida, en las limitaciones que introducen en el suministro de algunos servicios, como el del agua potable.

Finalmente hay que resaltar que esta actividad inmobiliaria se efectuó en torno a fraccionamientos residenciales campestres no ofreciéndose ninguno de carácter popular en el caso de los desarrollos estudiados.

\section{Bibliografía}

Fragoso, J.M., E. Concheiro y A. Gutiérrez (1979), El poder de la gran burguesía, Ediciones de Cultura Popular, México.

Gobierno del estado de Querétaro (1978), Cartografia de Querétaro, Gobierno del estado de Querétaro, Querétaro.

Gobierno del estado de Querétaro (1980), Plan Municipal de Desarrollo Urbano de Querétaro y Subregión Querétaro, Gobierno del estado de Querétaro, Querétaro.

Gravejat, A. (1970), La rente, le profit et la ville, Anthropos, París.

Guigou, J.L. (1982), "La rente fonciére. Les theories et leur évolution depuis 1960", Economica, París.

Krifa, H. (1979), "Le débat Topalov-Lipietz", en Revue d'Économie Régionale et Urbaine, 2, 214-235.

Legorreta, Jorge (1983), El proceso de urbanización en las ciudades petroleras, Centro de Ecodesarrollo, México.

SAHOP (1979), Plan de Desarrollo Urbano de Querétaro, SAHOP, México. 
Schteingart, Martha (1979), "Sector inmobiliario capitalista y formas de apropiación del suelo urbano: el caso de México", en Demografía y Economía, vol. XIII, núm. 4, 449-466.

Schteingart, Martha (1983), "La promoción inmobiliaria en el área metropolitana de la ciudad de México (1960-1980)', en Demografía y Economía, vol. XVII, núm. 53.

Super, John (1983), La vida en Querétaro durante la Colonia (1531-1810), FCE, México.

Topalov, Christian (1974), Les prometeurs immobiliers. Contribution à l'analyse de la production capitaliste de logement en France, Mouton, París.

Topalov, Christian (1975), Los agentes urbanos y la producción de vivienda, $\mathrm{Pu}$ blicaciones del Departamento de Geografía de la Universidad Autónoma de Barcelona, Bellaterra.

Topalov, Christian (1979), La urbanización capitalista, Edicol, México. 
